\title{
Prevalence of Thyroid Dysfunction in a Chinese Population with Different Glucose Intolerance Status: A Community-Based Cross-Sectional Study
}

This article was published in the following Dove Press journal:

Diabetes, Metabolic Syndrome and Obesity: Targets and Therapy

\author{
Xiuting Huang ${ }^{1, *}$ \\ Xiuying Zhang ${ }^{1} *$ \\ Xianghai Zhou (D) \\ Xueyao Han' \\ Zuodi $\mathrm{Fu}^{2}$ \\ Yufeng $\mathrm{Li}^{2}$ \\ Linong $\mathrm{Ji}^{1}$
}

\begin{abstract}
'Department of Endocrinology and Metabolism, Peking University People's Hospital, Peking University Diabetes Center, Beijing 100044, People's Republic of China; ${ }^{2}$ Department of Endocrinology and Metabolism, Capital Medical University Pinggu Teaching Hospital, Beijing 101200, People's Republic of China
\end{abstract}

*These authors contributed equally to this work

Correspondence: Yufeng Li

Department of Endocrinology and Metabolism, Capital Medical University

Pinggu Teaching Hospital, No. 59, Xinping North Street, Pinggu District, Beijing

101200, People's Republic of China

$\mathrm{Tel} / \mathrm{Fax}+861089978790$

Email doctorlyf@।26.com

Linong Ji

Department of Endocrinology and Metabolism, Peking University People's

Hospital, Peking University Diabetes

Center, No. II, Xizhimen South Street.

Xicheng District, Beijing 100044, People's

Republic of China

Tel +86 1088324108

$\mathrm{Fax}+861088325534$

Email jilinong@gmail.com
Aim: Impaired glucose metabolism and thyroid dysfunction (TD) are the two most common chronic metabolic disorders. This study aimed to investigate the epidemiological characteristics of TD in different status of glucose tolerance in a community-based Chinese population and to understand the association between TD and glucose metabolism.

Methods: A community-based population study of metabolic disease was conducted from June 2013 to September 2014 in Beijing, China. Residents aged 26-76 years were selected according to gender and age composition using multi-stage stratified random sampling process. All participants underwent serum thyroid function and thyroid-associated antibody tests. The status of glucose tolerance was determined using $75 \mathrm{~g}$-oral glucose tolerance test. Chi-square test was used to compare the differences in prevalence. Multivariate logistic regression analysis was used to determine the impact of insulin resistance (IR) on thyroid function.

Results: By analyzing 3986 participants who were included in the survey, the prevalence of type 2 diabetes (T2DM) and pre-diabetes (pre-DM) was $18.59 \%$ and $26.79 \%$, respectively. The prevalence of TD was $8.81 \%$, with overt hyperthyroidism accounting for $0.38 \%$; subclinical hyperthyroidism, $1.86 \%$; overt hypothyroidism, $0.70 \%$; and subclinical hypothyroidism, $5.87 \%$. The prevalence of TD increased with gradually deteriorated glucose tolerance (7.63\% in those with normal glucose tolerance, $9.27 \%$ in pre-DM, and $11.61 \%$ in T2DM) in both men and women. Each unit of higher HOMA-IR was associated with 7\% higher likelihood of having subclinical hypothyroidism.

Conclusion: The coexisting of TD with T2DM and pre-DM is high in this communitybased Chinese population, suggesting a close relationship between TD and glucose metabolism.

Keywords: type 2 diabetes, pre-diabetes, thyroid dysfunction, epidemiology

\section{Introduction}

Impaired glucose metabolism and thyroid disease are the two most common chronic endocrine disorders with a substantial overlap in the clinical setting. ${ }^{1-3}$ According to the latest report, approximately 463 million adults aged 20-79 years old worldwide suffer from diabetes in 2019; it is estimated that by 2030, diabetic patients will reach 578.4 million. ${ }^{4}$ The worldwide prevalence of diabetes is increasing, with studies showing higher prevalence in Asia. In particular, the prevalence of diabetes in China 
has increased by up to $11.6 \%$ in the latest national survey. ${ }^{5}$ Concurrently, the prevalence and spectrum of thyroid disorders have also increased, particularly those of clinical hypothyroidism and subclinical hypothyroidism. ${ }^{6,7}$ Thyroid hormones play key roles in all major metabolic pathways, including protein, carbohydrate, and lipid metabolism. However, previous studies have mostly focused on the relationship between type 2 diabetes mellitus (T2DM) and thyroid dysfunction (TD), while data on the association between the progress of TD and the natural course of T2DM are limited. Moreover, prevalence reports are often limited to one type of thyroid disease in patients with diabetes, and no study on the prevalence of various thyroid abnormalities under different glucose tolerance status in the general population has been conducted to date.

Recently, several epidemiological studies have shown a higher prevalence of hypothyroidism in patients with T2DM than that in the general population. ${ }^{8-11}$ An undiagnosed thyroid disorder may adversely affect metabolic control and increase the risk to an already predisposing T2DM. However, most of these studies analyzed a hospital-based population, a physical examination population, or an elderly population.

To address these issues, we conducted a study of nondiabetic patients and determined their glucose metabolism status using a 75 g-oral glucose tolerance test (OGTT) and evaluated thyroid dysfunction based on thyroid function tests in a large-scale community-based population in northern China. This study aimed to estimate the distribution of thyroid function disorders (ie, hyperthyroidism, subclinical hyperthyroidism, hypothyroidism, and subclinical hypothyroidism) in different glucose tolerance status (ie, T2DM, pre-diabetes mellitus (pre-DM), and normal glucose tolerance (NGT)) in the adult population. Our findings can serve as basis to further explore the relationship between glucose metabolism and thyroid disease, the interaction between their pathogenesis, and the appropriate screening and treatment strategies.

\section{Materials and Methods}

\section{Study Population}

The Pinggu Metabolic Disease Study (PMDS) was a community-based population study aimed to explore the natural course and pathogenesis of chronic metabolic diseases in the Chinese population. The PMDS recruited 6583 participants from the Pinggu district in Beijing, China. Patients were recruited between June 2013 and
September 2014 after meeting the following inclusion criteria: born in the Pinggu district, age 26-76 years, nonpregnant women, and residence in the district for at least 5 consecutive years at the start of the study. Participants were selected using a multi-stage random sampling process based on age and gender distribution of the population in the district. Finally, 4002 participants were selected and responded in this study. Those with incomplete data on thyroid function and blood glucose and those with severe liver and renal dysfunction were excluded. In this study, the population sample included 3986 participants.

This study was approved by the Ethics Committee of Peking University Health Science Center, and all participants provided written informed consent.

\section{Data Collection}

We collected all data using questionnaires via face-to-face field surveys. Demographic and anthropometric data included age, gender, height, body weight, body mass index (BMI), waist circumference, hip circumference, smoking status, educational levels, work status, etc. Blood pressure was measured using a sphygmomanometer (Omron, Japan) after resting for at least $10 \mathrm{~min}$ by trained staff according to standardized protocols. Weight and height were measured to calculate BMI by the formula: weight $(\mathrm{kg}) /$ height $^{2}\left(\mathrm{~m}^{2}\right)$. The clinical indicators were measured using standardized protocols by trained staff.

Fasting blood and urine samples from participants were collected after 8-hour fasting. Participants without known diabetes underwent a 75g 2-h oral glucose tolerance test (OGTT) to evaluate the status of glucose tolerance and those with diabetes had fasting plasma glucose measured. The laboratory measures included fasting and postprandial blood glucose levels, thyroid function and thyroid-related antibodies. All of the clinical samples collected from the participants were tested and analyzed in the laboratory of Peking University People's Hospital (Beijing, China) using standardized facilities and machines. All laboratory measures met the clinical diagnostic criteria. Plasma glucose was measured by hexokinase method. Hemoglobin A1c (HbA1c) was measured by cation-exchange highpressure liquid chromatography (HPLC) method (Adams A1c HA-8160; Arkray, Kyoto, Japan) which aligned to the Diabetes Control and Complications Trial (DCCT) standards. Fasting serum Alanine aminotransferase (ALT), Aspartate aminotransferase (AST), total cholesterol (TC), triglyceride (TG), high-density lipoprotein cholesterol (HDL-c), low-density lipoprotein cholesterol (LDL-c), 
glucose, and creatinine (CRE) levels and the uric acid (UA) were measured using the automated biochemical instrument (Coulter UniCel DxC 800, Beckman, Miami, FL, USA).

The thyroid hormones were measured via supersensitive electrochemiluminescence immunoassay (Chemilumi ACS-TSH, Siemens Healthcare Diagnostics K.K.). The investigation and the results of literature ${ }^{6}$ show that Beijing is a non-deficient area of iodine content. The Pinggu population is considered to have sufficient iodine intake. Combined with reference literature ${ }^{6}$ and the reference range of laboratory tests of our hospital, the reference range for serum thyroid-stimulating hormone (TSH) was set at $0.55-4.78 \mu \mathrm{IU} / \mathrm{mL}$ (limit of detection: $0.001 \mu \mathrm{IU} / \mathrm{mL}$ ), free thyroxine (FT4) was set at 11.45 $23.17 \mathrm{pmol} / \mathrm{L}$ and free triiodothyronine (FT3) was set at $3.50-6.50 \mathrm{pmol} / \mathrm{L}$, thyroid peroxidase antibodies (TPOAb) was set below $60 \mathrm{IU} / \mathrm{mL}$ and thyroglobulin antibody (TGAb) was set below $15 \mathrm{IU} / \mathrm{mL}$, respectively. The intraassay coefficient of variation (CV) was less than $8 \%$, and the inter-assay CV was less than $10 \%$ for all these parameters.

\section{Definitions}

NGT was defined as: fasting plasma glucose $(\mathrm{FPG})<6.1$ $\mathrm{mmol} / \mathrm{L}$ and 75g OGTT 2-h plasma glucose $(2-\mathrm{hPG})<7.8$ $\mathrm{mmol} / \mathrm{L}$; impaired glucose tolerance (IGT), FPG $<7.0$ $\mathrm{mmol} / \mathrm{L}$ and $7.8 \leq 2-\mathrm{hPG}<11.1 \mathrm{mmol} / \mathrm{L}$; and impaired fasting glucose (IFG), $6.1 \leq \mathrm{FPG}<7.0 \mathrm{mmol} / \mathrm{L}$ and $2-\mathrm{hPG}$ $<7.8 \mathrm{mmol} / \mathrm{L}$. Pre-DM included both IGT and IFG. A participant was defined to have diabetes if the FPG was $\geq 7.0 \mathrm{mmol} / \mathrm{L}$ or $2-\mathrm{hPG}$ was $\geq 11.1 \mathrm{mmol} / \mathrm{L}$, or there is a self-reported history of diabetes diagnosed by a doctor, and/or using anti-diabetes medication. Insulin resistance (IR) was estimated using homeostasis model assessment of IR $(\mathrm{HOMA}-\mathrm{IR}=\mathrm{FPG}, \mathrm{mmol} / \mathrm{L} \times$ fasting plasma insulin, $\mu \mathrm{U} / \mathrm{mL} / 22.5)$.

Thyroid function status was defined as follows: (1) normal thyroid function: $0.55 \leq \mathrm{TSH} \leq 4.78 \mu \mathrm{IU} / \mathrm{mL}$; (2) hyperthyroidism: TSH $<0.55 \mu \mathrm{IU} / \mathrm{mL}$ and FT4 $>23.17$ $\mathrm{pmol} / \mathrm{L}$ and/or $\mathrm{FT3}>6.5 \mathrm{pmol} / \mathrm{L}$; (3) subclinical hyperthyroidism: TSH $<0.55 \mu \mathrm{IU} / \mathrm{mL}$, and $11.45 \mathrm{pmol} / \mathrm{L}$ $<$ FT4 $<23.17 \mathrm{pmol} / \mathrm{L}$, and $3.5 \mathrm{pmol} / \mathrm{L}<\mathrm{FT} 3<6.5 \mathrm{pmol} /$ L; (4) hypothyroidism: TSH $>4.78 \mu \mathrm{IU} / \mathrm{mL}$ and FT4 $<$ $11.45 \mathrm{pmol} / \mathrm{L}$; (5) subclinical hypothyroidism: TSH $>4.78$ $\mu \mathrm{IU} / \mathrm{mL}$, and $11.45 \mathrm{pmol} / \mathrm{L}<\mathrm{FT} 4<23.17 \mathrm{pmol} / \mathrm{L}$.

\section{Statistical Analysis}

Categorical data were presented as number (\%). Separate analyses were conducted for men and women under different glucose tolerance status. Categorical variables were calculated by Chi-square $\left(X^{2}\right)$ test for comparison between women and men groups. Non-parametric statistical tests were used to compare the observed proportions between study groups. To evaluate the possible association of IR with subclinical hypothyroidism, a multivariate logistic regression was fitted with age, gender, BMI, and TPOAb as covariates. Separate regression model was also fitted by using the age and BMI categories, instead of continuous measures of these variables. The estimates of the confidence intervals of odds ratios were calculated. $\mathrm{P}<0.05$ was considered statistically significant. All statistical analyses were performed using SPSS (Chicago, IL, USA) version 20.0.

\section{Result}

\section{Characteristics of the Study Population}

Among the 4002 participants who answered the survey, 3986 were included in the analysis. The characteristic of the participants is shown in Table 1. The cohort comprised 1952 (48.97\%) men and 2034 (51.03\%) women, with 1799 (45.13\%) living in urban area and $2187(54.87 \%)$ in rural area. The mean age of the study population was 50.26 years, with a standard deviation (SD) of 11.76 years. The survey population was grouped by age according to a 5 -year range. The gender distribution in both the urban and rural areas in all age groups was similar. Given the different prevalence of diabetes and TD between women and men, we divided the participants by gender in the subsequent analyses.

\section{Distribution of Blood Glucose Status}

The overall prevalence of T2DM and pre-DM in this study was $18.59 \%$ and $26.79 \%$, respectively. The prevalence of T2DM and pre-DM in men was $20.95 \%$ and $26.95 \%$, in women was $16.32 \%$ and $26.65 \%$, respectively. The distribution of the three status of glucose metabolism in men and women was statistically different $\left(\chi^{2}=15.954, \mathrm{P}<0.001\right)$ (Table 2).

\section{Distribution of Thyroid Function Status}

According to the above definition of thyroid-related diseases, 351 participants had abnormal thyroid function. The overall prevalence of TD in this community population was approximately $8.81 \%$, with overt hyperthyroidism accounting for $0.38 \%$; subclinical 
Table I Characteristics of the Participants

\begin{tabular}{|c|c|c|c|c|c|c|}
\hline Variables & Characteristic & Mean & SD & Median & 25th & 75th \\
\hline Total number & 3986 & 1 & 1 & 1 & 1 & l \\
\hline Gender, male, No. (\%) & $1952(48.97)$ & 1 & l & l & 1 & 1 \\
\hline Female, No. (\%) & $2034(51.03)$ & 1 & 1 & 1 & 1 & 1 \\
\hline Age, Mean(SD), years & $50.26 \pm 11.76$ & 50.26 & 11.76 & 50.53 & 41.59 & 59.04 \\
\hline BMI, Mean(SD), kg.m ${ }^{-2}$ & $26.11 \pm 3.83$ & 26.11 & 3.83 & 25.85 & 23.42 & 28.37 \\
\hline \multicolumn{7}{|l|}{ Waist circumference, Mean(SD), cm } \\
\hline Male & $89.35 \pm 10.23$ & 89.35 & 10.23 & 89.50 & 82.00 & 96.60 \\
\hline Female & $84.31 \pm 10.75$ & 84.31 & 10.75 & 84.00 & 76.80 & 91.50 \\
\hline SBP, Mean (SD), mmHg & $130.13 \pm 18.05$ & 130.13 & 18.05 & 128.33 & 117.33 & 140.67 \\
\hline DBP, Mean (SD), mmHg & $78.73 \pm 11.38$ & 78.73 & 11.38 & 78.00 & 71.00 & 85.67 \\
\hline FPG, Median (IQR), mmol/L & $5.64(5.27,6.22)$ & 6.08 & 1.63 & 5.64 & 5.27 & 6.22 \\
\hline 2h PG, Median (IQR), mmol/L & $6.91(5.81,8.31)$ & 7.39 & 2.58 & 6.91 & 5.81 & 8.31 \\
\hline HbAlc, Median (IQR), \% & $5.60(5.40,5.90)$ & 5.83 & 0.94 & 5.60 & 5.40 & 5.90 \\
\hline HbAlc, Median (IQR), $\mathrm{mmol} / \mathrm{mol}$ & $37.7 I(35.52,40.98)$ & 40.23 & 10.22 & 37.71 & 35.52 & 40.98 \\
\hline FINS, Median (IQR), uU/mL & $8.22(5.54,12.16)$ & 9.72 & 6.60 & 8.22 & 5.54 & 12.16 \\
\hline PINS, Median (IQR), uU/mL & $51.58(31.61,83.88)$ & 67.30 & 56.23 & 51.58 & 31.61 & 83.88 \\
\hline Triglycerides, Median (IQR), mmol/L & $1.22(0.78,1.89)$ & 1.59 & $\mathrm{I} .43$ & 1.22 & 0.78 & 1.89 \\
\hline LDL-C, Mean (SD), mmol/L & $2.88 \pm 0.81$ & 2.88 & 0.81 & 2.84 & 2.32 & 3.37 \\
\hline \multicolumn{7}{|l|}{ HDL-C, Mean (SD), mmol/L } \\
\hline Male & $1.11 \pm 0.32$ & 1.11 & 0.32 & 1.05 & 0.90 & 1.24 \\
\hline Female & $1.21 \pm 0.29$ & 1.21 & 0.29 & 1.17 & 1.01 & 1.36 \\
\hline ALT, Mean (SD), U/L & $23.81 \pm 18.58$ & 23.81 & 18.58 & 20.00 & 15.00 & 27.00 \\
\hline AST, Mean (SD), U/L & $23.14 \pm 11.43$ & 23.14 & 11.43 & 21.00 & 18.00 & 25.00 \\
\hline CRE, Mean (SD), umol/L & $60.82 \pm 14.66$ & 60.82 & 14.66 & 59.00 & 50.00 & 69.00 \\
\hline UA, Mean (SD), umol/L & $285.42 \pm 80.50$ & 285.42 & 80.50 & 276.00 & 228.00 & 333.00 \\
\hline TT4, Median (IQR), ug/dL & $8.50(7.50,9.60)$ & 8.54 & 1.64 & 8.50 & 7.50 & 9.60 \\
\hline FT4, Median (IQR), pmol/L & $15.99(\mid 4.58,17.59)$ & 16.17 & 2.50 & 15.99 & 14.58 & 17.59 \\
\hline TT3, Median (IQR), ng/dL & I 16.49(104.50, 128.44) & 117.49 & 19.78 & 116.49 & 104.50 & 128.44 \\
\hline FT3, Median (IQR), pmol/L & $4.97(4.63,5.34)$ & 5.00 & 0.70 & 4.97 & 4.63 & 5.34 \\
\hline TSH, Median (IQR), ulU/mL & $\mathrm{I} .90(\mathrm{I} .3 \mathrm{I}, 2.8 \mathrm{I})$ & 2.47 & 3.86 & 1.90 & 1.31 & 2.81 \\
\hline TPOAb, No. (\%), (+) & $532(13.35)$ & 1 & 1 & 1 & 1 & 1 \\
\hline TGAb, No. (\%), (+) & $480(12.04)$ & I & I & I & I & I \\
\hline
\end{tabular}

Notes: The data are presented as No. and \% for category variables; the data are presented as the means (SDs) or medians (interquartile ranges, IQRs) for continuous variables.

Abbreviations: SBP, systolic blood pressure; DBP, diastolic blood pressure; BMI, body mass index; FPG, fasting plasma glucose; 2h PG, 2-hour plasma glucose during 75goral glucose tolerance test (OGTT); HbAlc, hemoglobin AIc; FINS, fasting insulin; PINS, postprandial insulin; TC, total cholesterol; LDL-c, low-density lipoprotein cholesterol; HDL-c, high-density lipoprotein cholesterol; TG, triglyceride; UA, uric acid; CRE, serum creatinine; TSH, thyroid-stimulating hormone; FT4, free thyroxine; FT3, free triiodothyronine; TT4, total thyroxine; TT3, total triiodothyronine; TPOAb, thyroid peroxidase antibody; TGAb, thyroglobulin antibody.

hyperthyroidism, 1.86\%; overt hypothyroidism, $0.70 \%$; and subclinical hypothyroidism, $5.87 \%$ (Table 3 ). The prevalence of TD in men was significantly lower than that in women $\left(5.79 \%\right.$ vs $11.70 \%, \chi^{2}=43.356, \mathrm{P}<$ 0.001). As shown in Table 3, the proportion of different TD status was statistically different between men and women $\left(\chi^{2}=72.291, \mathrm{P}<0.001\right)$. Notably, subclinical hypothyroidism was the most common TD in both men $(3.13 \%)$ and women $(8.51 \%)$.
Table 2 The Distribution of Blood Glucose Status of the Sample by $\operatorname{Sex}\left(\chi^{2}\right.$ Test)

\begin{tabular}{|l|l|l|l|l|}
\hline & NGT & Pre-DM & T2DM & Total \\
\hline Men & $1017(52.10)$ & $526(26.95)$ & $409(20.95)$ & 1952 \\
Women & $1160(57.03)$ & $542(26.65)$ & $332(16.32)$ & 2034 \\
Total & $2177(54.62)$ & $1068(26.79)$ & $741(18.59)$ & 3986 \\
\hline
\end{tabular}

Notes: $\chi^{2}=15.954 . P<0.001$. The data are presented as No. and \%. P value is for the trend of across quartiles.

Abbreviations: NGT, normal glucose tolerance; Pre-DM, pre-diabetes; T2DM, type 2 diabetes mellitus. 
Table 3 The Distribution of Thyroid Function Status of the Sample by Sex $\left(\chi^{2}\right.$ Test)

\begin{tabular}{|c|c|c|c|c|c|c|}
\hline & $\begin{array}{l}\text { Overt } \\
\text { Hyperthyroidism }\end{array}$ & $\begin{array}{l}\text { Subclinical } \\
\text { Hyperthyroidism }\end{array}$ & $\begin{array}{l}\text { Overt } \\
\text { Hypothyroidism }\end{array}$ & $\begin{array}{l}\text { Subclinical } \\
\text { Hypothyroidism }\end{array}$ & $\begin{array}{l}\text { Normal Thyroid } \\
\text { Function }\end{array}$ & Total \\
\hline Men & $6(0.3 I)$ & $43(2.20)$ & $3(0.15)$ & $61(3.13)$ & $1839(94.21)$ & 1952 \\
\hline Women & $9(0.44)$ & 31 (1.52) & $25(1.23)$ & $173(8.51)$ & $1796(88.30)$ & 2034 \\
\hline Total & $15(0.38)$ & $74(1.86)$ & $28(0.70)$ & $234(5.87)$ & 3635 (91.19) & 3986 \\
\hline
\end{tabular}

Notes: $\chi^{2}=72.291$. $P<0.001$. The data are presented as No. and \%. P value is for the trend of across quartiles.

\section{Proportion of Thyroid Dysfunction in Different Status of Glucose Tolerance}

The proportion of overt and subclinical hyperthyroidism and hypothyroidism in men and women in different status of glucose metabolism is shown in Table 4. A total of $7.63 \%, 9.27 \%$, and $11.61 \%$ of participants had TD among those with NGT, pre-DM, and T2DM, respectively, with the rate of TD being higher in women than that in men. The proportions of various TDs were statistically different in the three status of glucose metabolism both in men $(\mathrm{P}=$ $0.028)$ and in women $(P=0.027)$.

\section{Distribution of Subclinical Hypothyroidism in Different Status of Glucose Tolerance by Gender}

As shown in Table 4, the proportion of subclinical hypothyroidism among men was $1.97 \%, 4.18 \%$, and $4.65 \%$ in those with NGT, pre-DM, and T2DM, respectively. Correspondingly, the proportion among women was $7.33 \%$, $9.04 \%$, and $11.75 \%$ in those with NGT, pre-DM, T2DM, respectively. Subclinical hypothyroidism was more common in women than in men in every status of glucose tolerance and tended to increase with worsening blood glucose tolerance.

\section{The Effect of Insulin Resistance on Thyroid Function}

Given the high prevalence of subclinical hypothyroidism in those with impaired glucose tolerance status, we evaluated the relationship between subclinical hypothyroidism and IR to identify the potential association between glucose metabolism and thyroid hormones.

Comparison analysis showed that the patients with subclinical hypothyroidism had higher HOMA-IR than those with normal thyroid function $[2.44(1.44,3.93)$ vs 2.12 (1.39, 3.26), $\mathrm{P}=0.018]$. Multivariate logistic regression analysis showed that the levels of IR were positively associated with subclinical hypothyroidism after adjusting for age, gender, BMI, and TPOAb. One unit higher IR was associated with $7 \%$ higher likelihood of having subclinical hypothyroidism (OR=1.07, 95\% CI: 1.03-1.12).

\section{Discussion}

In our study, we estimated the prevalence of various thyroid abnormalities under different glucose intolerance status in a large sample community-based population in northern China. The proportion of thyroid dysfunction was higher in subjects with impaired glucose tolerance than normal individuals. Thyroid dysfunction especially

Table 4 The Distribution of Thyroid Function Status in Different Glucose Tolerance Population by Sex

\begin{tabular}{|c|c|c|c|c|c|c|c|}
\hline & & $\begin{array}{l}\text { Overt } \\
\text { Hyperthyroidism }\end{array}$ & $\begin{array}{l}\text { Subclinical } \\
\text { Hyperthyroidism }\end{array}$ & $\begin{array}{l}\text { Overt } \\
\text { Hypothyroidism }\end{array}$ & $\begin{array}{l}\text { Subclinical } \\
\text { Hypothyroidism }\end{array}$ & $\begin{array}{l}\text { Normal Thyroid } \\
\text { Function }\end{array}$ & Total \\
\hline \multirow[t]{3}{*}{ NGT } & Men & $3(0.29)$ & $26(2.56)$ & $2(0.20)$ & $20(1.97)$ & 966 (94.99) & 1017 \\
\hline & Women & $5(0.43)$ & $12(1.03)$ & $13(1.12)$ & 85 (7.33) & 1045 (90.09) & 1160 \\
\hline & Total & $8(0.37)$ & 38 (I.75) & $15(0.69)$ & $105(4.82)$ & 2011 (92.37) & 2177 \\
\hline \multirow[t]{3}{*}{ Pre-DM } & Men & I (0.19) & $6(1.14)$ & I (0.19) & $22(4.18)$ & $496(94.30)$ & 526 \\
\hline & Women & $3(0.55)$ & 8 (I.48) & $9(1.66)$ & $49(9.04)$ & 473 (87.27) & 542 \\
\hline & Total & $4(0.37)$ & $14(1.31)$ & $10(0.94)$ & 7I (6.65) & $969(90.73)$ & 1068 \\
\hline \multirow[t]{3}{*}{ T2DM } & Men & $2(0.49)$ & II (2.69) & $0(0.00)$ & $19(4.65)$ & 377 (92.18) & 409 \\
\hline & Women & I $(0.30)$ & II (3.3I) & $3(0.90)$ & 39 (II.75) & 278 (83.73) & 332 \\
\hline & Total & $3(0.40)$ & $22(2.97)$ & $3(0.40)$ & $58(7.83)$ & 655 (88.39) & 741 \\
\hline
\end{tabular}

Note: The data are presented as No. and \%.

Abbreviations: NGT, normal glucose tolerance; Pre-DM, pre-diabetes; T2DM, type 2 diabetes mellitus. 
subclinical hypothyroidism was closely associated with deteriorating glucose metabolism. It was worth noting that IR index was significantly related to the development of subclinical hypothyroidism, and as the severity of insulin resistance increased, the possibility of subclinical hypothyroidism increased.

Many cross-sectional and cohort studies have investigated the coexistence of diabetes and thyroid disease, with the prevalence of thyroid disease varying from $10.8 \%$ to $32.4 \%$ in patients with diabetes. ${ }^{8,9,12-15}$ Radaideh et al reported an approximately $12.5 \%$ prevalence of thyroid disease in the patients with diabetes, which was significantly higher compared to the $6.6 \%$ in a control group of healthy subjects in a Jordan study. ${ }^{8}$ A systematic review and meta-analysis of multinational studies showed a higher prevalence of hypothyroidism in those with T2DM than in non-diabetic participants. ${ }^{10}$ In a Danish prospective cohort with a mean follow-up of 6 years, the hazard ratio (HR) of comorbidity with diabetes in patients with hypothyroidism was 1.40 (1.11-1.77) compared with those with normal thyroid function. ${ }^{16}$ However, Gholampour et al conducted a study within the framework of the Tehran Thyroid Study and reported that the incidence of TDs in T2DM or pre-DM was similar with that of healthy controls. ${ }^{17}$ The inconsistent results might be due to the differences in ethnicity, race, age, gender, iodine status, and the laboratory measurements.

Most of the above studies recruited participants from hospitals or medical diagnostic clinics. Moreover, these studies were mainly focus on thyroid dysfunction or impaired glucose tolerance status, respectively, such as focus on subclinical hypothyroidism or overt diabetes. Our study was based on a large and well-characterized community population that was randomly sampled according to gender, age compositions, and living area and represented a typical Chinese community. We not only observed significant difference in the prevalence of TD among the three status of glucose metabolism but also significant difference between genders. The current analysis showed that the incidence of subclinical hypothyroidism increased gradually with worsening glucose metabolism from NGT, pre-diabetes to diabetes, particularly in women.

However, the potential causal relationship between thyroid function and T2DM is still unclear and more complicated. There were several pathways that might explain the observed relation between TD and the risk of diabetes. Thyroid hormones regulated glucose homeostasis through various physiological mechanisms including intestinal glucose absorption, hepatic glucose secretion, cellular glucose transport, and the secretion of insulin and major counter-

regulatory hormones. ${ }^{3}$ In the liver, thyroid hormone could increase the expression of glucose 6-phosphate and phosphoenolpyruvate carboxykinase (PEPCK), an enzyme that enhances gluconeogenesis. Enhanced glycogenolysis and increased hepatic glucose output could cause hyperinsulinemia and glucose intolerance, leading to peripheral IR. ${ }^{18,19}$ In the gastrointestinal tract, thyroid hormones increase gastrointestinal motility and enhance glucose absorption. ${ }^{18}$ Thyroid hormones are important for normal pancreatic islet development. ${ }^{20}$ Besides, thyroid hormones exert profound effects on the regulation of lipid metabolism throughout both the central nervous system and the peripheral target organs. Thyroid hormones increase lipolysis in adipose tissue and increase the uptake of fatty acid by regulating fatty acid transporter protein and enhance hepatic lipogenesis in liver. Elevated serumfree fatty acid levels cause IR. ${ }^{18}$ Thyroid hormones modulate the synthesis, mobilization and breakdown of cholesterol. Hypothyroidism is characterized by hypercholesterolaemia, higher levels of LDL-c levels, $\mathrm{TG}$, and apolipoprotein $\mathrm{B}$ levels because of a decreased fractional clearance of LDL-c by a reduced number of LDL receptors in liver. ${ }^{21,22}$ On the other hand, hyperglycemia affects thyroid function by changing the level of TSH and impairing the conversion of thyroxine (T4) to triiodothyroxine (T3) in the peripheral tissues. There is an association between duration of diabetes and hypothyroidism, which suggests that a long-lasting hyperglycemia might have a cumulative effect on TD. ${ }^{23,24}$

In addition, inflammation might be involved in the pathogenesis of both thyroid diseases and diabetes. High levels of inflammatory cytokines in those with diabetes might accelerate hypothyroidism. ${ }^{25-28}$ Studies have shown that in the condition of abnormal thyroid function (both hyper- and hypothyroidism), the levels of TNF- $\alpha$ in tissues and systemic circulation could be increased. Importantly, TNF- $\alpha$ levels were positively associated with IR and fasting blood glucose. ${ }^{29-31}$ Concurrently, the increase of inflammatory factors in abnormal thyroid function might be involved in the onset of diabetes. However, the exact pathophysiological mechanisms through which thyroid function could affect the risk of diabetes in the general population remain to be determined. 
Recently, the high prevalence of subclinical hypothyroidism and its pathophysiology have received substantial research attention. In our study, the patients with subclinical hypothyroidism had higher IR index than those with normal thyroid function. The treatment of hypothyroidism has been shown to restore insulin sensitivity and the secretion of glucoregulatory hormones. ${ }^{32}$ The coexistence of hypothyroidism with T2DM might accelerate the progression of metabolic disease. ${ }^{33}$ However, the phenomenon of IR increasing the risk of subclinical hypothyroidism needs further research to clarify the underlying mechanisms.

This study included the relatively large sample size taken from a community-based population and the detailed information on demography, physical examination, medical history, and laboratory indicators. These allowed for relatively reliable evaluations of the association of interest and generalizability of the findings. Both TD and T2DM require long-term continuous treatment, it is important to screen for thyroid function in glucose intolerance patients as their complex interdependent interactions. The multifaceted relationship between thyroid dysfunctions and blood glucose disorders had implications for the clinician. However, the cross-sectional design of the study limited our ability to infer causal relationships between variations in thyroid dysfunction and impaired glucose metabolism. More cohort study and/or experimental studies are needed to clarify the relationship between the two diseases and their appropriate treatment strategies.

\section{Conclusions}

In conclusion, this study quantified the prevalence of thyroid dysfunction under different status of glucose tolerance based on a community-based population in northern China and showed that thyroid dysfunction especially subclinical hypothyroidism is closely associated with glucose metabolism.

\section{Ethical Approval and Informed Consent}

This study was approved by the Ethics Committee of Peking University Health Science Center, and all participants provided written informed consent. All procedures in the study were performed in accordance with the ethical standards of the Declaration of Helsinki.

\section{Acknowledgments}

We thank all research staff for their collection of data. We also thank all the study participants for their contributions.
Co-corresponding authors Linong $\mathrm{Ji}$ and Yufeng $\mathrm{Li}$ contributed equally to this work

\section{Funding}

This work was supported through a grant from Beijing Science and Technology Committee (D131100005313008). This project was also supported by NIH research training grant R25 TW009345 funded by the Fogarty International Center. We thank all the participants for agreeing to join this study. We are grateful to the research teams from the endocrinology and metabolic department of Beijing Pinggu Hospital and Peking University People's hospital for their contribution to field survey and data collection.

\section{Disclosure}

The authors report no conflicts of interest for this work and declare that there is no conflict of interest regarding the publication of this article.

\section{References}

1. Canaris GJ, Manowitz NR, Mayor G, Ridgway EC. The Colorado thyroid disease prevalence study. Arch Intern Med. 2000;160 (4):526-534. doi:10.1001/archinte.160.4.526

2. Kadiyala R, Peter R, Okosieme OE. Thyroid dysfunction in patients with diabetes: clinical implications and screening strategies. Int $J$ Clin Pract. 2010;64(8):1130-1139. doi:10.1111/j.17421241.2010.02376.x

3. Duntas LH, Orgiazzi J, Brabant G. The interface between thyroid and diabetes mellitus. Clin Endocrinol (Oxf). 2011;75(1):1-9. doi:10.1111/j.1365-2265.2011.04029.x

4. IDF. Diabetes atlas - 9th edition; 2019. Available from: www.diabe tesatlas.org. Accessed November 3, 2020.

5. Xu Y, Wang L, He J, et al. Prevalence and control of diabetes in Chinese adults. JAMA. 2013;310(9):948-959. doi:10.1001/ jama.2013.168118

6. Shan Z, Chen L, Lian X, et al. Iodine status and prevalence of thyroid disorders after introduction of mandatory universal salt iodization for 16 years in China: a cross-sectional study in 10 cities. Thyroid. 2016;26(8):1125-1130. doi:10.1089/thy.2015.0613

7. Hollowell JG, Staehling NW, Flanders WD, et al. Serum TSH, T(4), and thyroid antibodies in the United States population (1988 to 1994): national health and nutrition examination survey (NHANES III). J Clin Endocrinol Metab. 2002;87(2):489-499.

8. Radaideh AR, Nusier MK, Amari FL, et al. Thyroid dysfunction in patients with type 2 diabetes mellitus in Jordan. Saudi Med J. 2004;25(8):1046-1050.

9. Perros P, McCrimmon RJ, Shaw G, Frier BM. Frequency of thyroid dysfunction in diabetic patients: value of annual screening. Diabet Med. 1995;12(7):622-627. doi:10.1111/j.1464-5491.1995.tb00553.x

10. Han C, He X, Xia X, et al. Subclinical hypothyroidism and type 2 diabetes: a systematic review and meta-analysis. PLoS One. 2015;10 (8): $\mathrm{e} 0135233$.

11. Gronich N, Deftereos SN, Lavi I, Persidis AS, Abernethy DR, Rennert G. Hypothyroidism is a risk factor for new-onset diabetes: a cohort study. Diabetes Care. 2015;38(9):1657-1664. doi:10.2337/ dc14-2515 
12. Smithson MJ. Screening for thyroid dysfunction in a community population of diabetic patients. Diabet Med. 1998;15(2):148-150. doi:10.1002/(SICI)1096-9136(199802)15:2<148::AID-DIA540>3.0. $\mathrm{CO} ; 2-\mathrm{H}$

13. Palma CC, Pavesi M, Nogueira VG, et al. Prevalence of thyroid dysfunction in patients with diabetes mellitus. Diabetol Metab Syndr. 2013;5(1):58. doi:10.1186/1758-5996-5-58

14. Diez JJ, Sanchez P, Iglesias P. Prevalence of thyroid dysfunction in patients with type 2 diabetes. Exp Clin Endocrinol Diabetes. 2011;119(4):201-207. doi:10.1055/s-0031-1271691

15. Al-Geffari M, Ahmad NA, Al-Sharqawi AH, Youssef AM, Alnaqeb D, Al-Rubeaan K. Risk factors for thyroid dysfunction among type 2 diabetic patients in a highly diabetes mellitus prevalent society. Int $J$ Endocrinol. 2013;2013:417920. doi:10.1155/2013/ 417920

16. Thvilum M, Brandt F, Almind D, Christensen K, Brix TH, Hegedus L. Type and extent of somatic morbidity before and after the diagnosis of hypothyroidism. a nationwide register study. PLoS One. 2013;8(9):e75789. doi:10.1371/journal.pone.0075789

17. Gholampour Dehaki M, Amouzegar A, Delshad H, Mehrabi Y, Tohidi M, Azizi F. Thyroid dysfunction in patients with impaired glucose metabolism: 11 year follow up from the Tehran thyroid study. PLoS One. 2017;12(10):e0184808. doi:10.1371/journal.pone.0184808

18. Nishi M. Diabetes mellitus and thyroid diseases. Diabetol Int. 2018;9 (2):108-112.

19. Wang C. The relationship between type 2 diabetes mellitus and related thyroid diseases. J Diabetes Res. 2013;2013:390534. doi: $10.1155 / 2013 / 390534$

20. Fukuchi M, Shimabukuro M, Shimajiri Y, et al. Evidence for a deficient pancreatic beta-cell response in a rat model of hyperthyroidism. Life Sci. 2002;71(9):1059-1070. doi:10.1016/ S0024-3205(02)01791-5

21. Ritter MJ, Amano I, Hollenberg AN. Thyroid hormone signaling and the liver. Hepatology. 2020;72(2):742-752. doi:10.1002/hep.31296

22. Duntas LH. Thyroid disease and lipids. Thyroid. 2002;12 (4):287-293.

23. Nair A, Jayakumari C, Jabbar PK, et al. Prevalence and associations of hypothyroidism in indian patients with type 2 diabetes mellitus. J Thyroid Res. 2018;2018:5386129. doi:10.1155/2018/5386129
24. Kalra S, Aggarwal S, Khandelwal D. Thyroid dysfunction and type 2 diabetes mellitus: screening strategies and implications for management. Diabetes Ther. 2019;10(6):2035-2044. doi:10.1007/ s13300-019-00700-4

25. Mirza S, Hossain M, Mathews C, et al. Type 2-diabetes is associated with elevated levels of TNF-alpha, IL-6 and adiponectin and low levels of leptin in a population of Mexican Americans: a cross-sectional study. Cytokine. 2012;57(1):136-142.

26. Lu L, Zhang Q, Pu LJ, et al. Elevation of tumor necrosis factor-alpha, interleukin-1beta and interleukin-6 levels in aortic intima of Chinese Guizhou minipigs with streptozotocin-induced diabetes. Chin Med J. 2007;120(6):479-484. doi:10.1097/00029330-200703020-00009

27. Zakarija M, McKenzie JM. Influence of cytokines on growth and differentiated function of FRTL5 cells. Endocrinology. 1989;125 (3):1260-1265. doi:10.1210/endo-125-3-1260

28. Molnar I, Balazs C, Szegedi G, Sipka S. Inhibition of type 2,5'deiodinase by tumor necrosis factor alpha, interleukin- 6 and interferon gamma in human thyroid tissue. Immunol Lett. 2002;80(1):3-7. doi:10.1016/S0165-2478(01)00301-7

29. Aust G, Heuer M, Laue S, et al. Expression of tumour necrosis factor-alpha (TNF-alpha) mRNA and protein in pathological thyroid tissue and carcinoma cell lines. Clin Exp Immunol. 1996;105 (1):148-154. doi:10.1046/j.1365-2249.1996.d01-726.x

30. Diez JJ, Hernanz A, Medina S, Bayon C, Iglesias P. Serum concentrations of tumour necrosis factor-alpha (TNF-alpha) and soluble TNF-alpha receptor p55 in patients with hypothyroidism and hyperthyroidism before and after normalization of thyroid function. Clin Endocrinol (Oxf). 2002;57(4):515-521.

31. Nilsson J, Jovinge S, Niemann A, Reneland R, Lithell H. Relation between plasma tumor necrosis factor-alpha and insulin sensitivity in elderly men with non-insulin-dependent diabetes mellitus. Arterioscler Thromb Vasc Biol. 1998;18(8):1199-1202. doi:10.1161/ 01.ATV.18.8.1199

32. Stanicka S, Vondra K, Pelikanova T, Vlcek P, Hill M, Zamrazil V. Insulin sensitivity and counter-regulatory hormones in hypothyroidism and during thyroid hormone replacement therapy. Clin Chem Lab Med. 2005;43(7):715-720. doi:10.1515/CCLM.2005.121

33. Volzke H, Ludemann J, Robinson DM, et al. The prevalence of undiagnosed thyroid disorders in a previously iodine-deficient area. Thyroid. 2003;13(8):803-810. doi:10.1089/105072503768499680

\section{Publish your work in this journal}

Diabetes, Metabolic Syndrome and Obesity: Targets and Therapy is an international, peer-reviewed open-access journal committed to the rapid publication of the latest laboratory and clinical findings in the fields of diabetes, metabolic syndrome and obesity research. Original research, review, case reports, hypothesis formation, expert opinion and commentaries are all considered for publication. The manuscript management system is completely online and includes a very quick and fair peer-review system, which is all easy to use. Visit http://www.dovepress.com/testimonials.php to read real quotes from published authors. 